\title{
Círculo virTuoso de LA EDUCACIÓN EMPRESARIAL: UNA PROPUESTA DE MODELO PARA EL DESARROLLO DE UN TEJIDO EMPRESARIAL DE CALIDAD
}

\section{Resumen}

En este artículo se presentan avances de investigación científica referente al tema de la educación empresarial como ente detonador de la creación de empresas de los denominados EBU, Empresarios de Base Universitaria. Así mismo, presenta una propuesta de modelo de desarrollo económico basado en la educación empresarial como ente generador de un tejido empresarial de calidad. El objetivo principal es aportar a la investigación científica en el tema de la creación de empresas en el marco de la teoría Institucional de North, específicamente en países con economías emergentes como Colombia y en donde no se han realizado muchos avances investigativos desde lo científico (Bruton, Ahlstrom, Obloj 2008). Los objetivos específicos que presenta el artículo incluyen la presentación de algunos avances de la investigación empírica paor parte del autor de la definición de tejido empresarial de calidad y educación empresarial basado en los estudios de Santos y Liñán (2005), además de la revisión bibliográfica principal del ET\&P Entrepreneurship Theory and Practica Journal.

\section{Palabras clave}

Espíritu empresarial Formación empresarial Educación empresarial Desarrollo económico

Tejido empresarial Creación de empresas Pedagogía del emprendimiento.
John Orlando Crissien Castillo*

\section{INTRODUCCIÓN}

\section{Tejido empresarial}

Más empresarios; suficientes empresarios. Más empresas; suficientes empresas. Mejores empresarios; buenos empresarios que creen mejores empresas, suficientes buenas empresas que soporten el aparato productivo del país y así generar desarrollo económico. Este es el tejido empresarial que genera economías empresariales éticas y en esencia el desarrollo económico ético.

La calidad del tejido empresarial y la calidad de los empresarios de un país, los cuales de manera ética aprovechan y crean recursos tradicionales y no tradicionales, se logra con un sistema de creencias que privilegia el espíritu empresarial a diferencia del espíritu empleacional.

¿Cómo se logra desarrollar un sistema de creencias nuevo en la población? ¿Cómo

\footnotetext{
* John Crissien. Doctorando en Ciencias Empresariales de la Universidad Antonio de Nebrija España, MBA, Master of Business Administration, WIU. EEUU, MAD Master en Alta Dirección, Colegio de Graduados en Alta Direcion, Mexico D.F. Administrador de Empresas EAN, Colombia. Consejero-Profesor Asociado facultad de posgrados. Universidad EAN.
} 
se logra despertar el espíritu empresarial en los individuos? La respuesta es sencilla. Mediante la educación empresarial.

En este artículo se abarcan los tópicos anteriores descritos. Primero el desarrollo económico de un país como fuente de bienestar y la calidad de vida. La formación de un tejido empresarial de calidad como base del mencionado desarrollo económico y finalmente la educación empresarial como detonador de dicho tejido. A diferencia de teorías que se basan en el perfil y las características de personalidad de los empresarios ( Mcmillan) o teorías que basan la creación de nuevas empresas en el soporte que el gobierno brinda a los nuevos emprendedores. Este artículo se fundamenta en la universidad como ese detonador, la universidad como esa institución que brinda la formación y el apoyo para que los estudiantes creen nuevas empresas y fortalezcan el tejido empresarial.

Para esto, la institución debe tomar una nueva responsabilidad y tornarse de una Universidad como institución alejada de la empresa, alejada del desarrollo económico, a ser una institución responsable de este, mediante su cambio de orientación en la formación. Una educación empresarial. Como conclusión de este artículo se presentarán los resultados de la revisión bibliográfica en el tema y de manera empírica se presenta una propuesta de clasificación de universidades con educación empresarial. Muchas puertas se abren para la investigación científica en Colombia y Latinoamérica en este tema.

\section{Abstract}

This is an article that shows the progress of a scientific research based on entrepreneurial education as the bases for company creation of the well known EBU, University Professional Managers (Calixto et al 2004). Similarly, this papers presents the proposal of an economic development model based on managerial education as the principal agent of DE quality entrepreneurial network. It aims at giving a great impulse to scientific research based on company creation, under the lights of North's institutional theory , specifically in underdeveloped and developing economies, in which small advances and few studies have been made (Bruton, Ahlstrom, Obloj 2008) The author of this article also presents some scientific empirical considerations on the quality entrepreneurial network and managerial education based on the studies of Santos and Liñán (2005),

besides making a bibliographic revision of ET \& P, Entrepreneurship

Theory and Practical Journal.

\section{KEY WORDS}

Entrepreneurial spirit

Managerial formation

Economic development

Entrepreneurial network

Company creation

Entrepreneurship pedagogy 


\section{Educación EMPRESARIAL COMO DETONADOR DEL TEJIDO EMPRESARIAL DE CALIDAD}

\section{$\mathbf{L}_{\text {a educacion empressaral }}$} genera en los individuos espíritu empresarial, que es esa fuerza interna que direcciona a los estudiantes a ser empresarios; se generan empresarios que crean empresas. Muchos individuos con el mismo sistema de pensamiento generan cultura empresarial. Se crean empresas. para crecer se necesita que estos empresarios mejoren, que sean buenos empresarios que con las empresas que crearon en un inicio se fortalezcan y se desarrollen para ser buenas empresas (que generen valor agregado a la economía no solo empresas de supervivencia para el empresario); suficientes buenos empresarios que crean y desarrollan buenas empresas, crean un bosque de buenas empresas y empresarios que aportan al desarrollo económico y generan cambios en la cultura de una nación, una nación de empresarios, en donde como círculo virtuoso los modelos de rol para los jóvenes será el ser empresario. El círculo vicioso es el que vivimos hoy, es mal visto entre los jóvenes el pensar en ser empresario, esto porque hay que ser "Doctor" $y$ trabajar en una multinacional o en un banco, para ser alguien en la vida.

Un tejido empresarial de calidad es aquel en donde las empresas nacionales se fortalecen y pasan de ser buenas empresas, ya sean pequeñas o medianas, a ser empresas multinacionales aportando al PIB y a la generación de empleo y competitividad internacional del país.
Un tejido empresarial débil o de poca calidad es el conformado por pequeñas empresas que no evolucionan y no crecen; ante cualquier turbulencia internacional el país se convulsiona y estas empresas son muy vulnerables, no solo por la deficiencia en gerencia profesional sino también por su falta de competitividad; así mismo parte de este tejido empresarial son las empresas multinacionales que por interés del mercado o atractivos impositivos penetran el país como Inversión extranjera directa y ante cualquier cambio de políticas o de intereses organizacionales dejan el país desarticulando fuertemente el tejido empresarial y por debilitando el desarrollo económico.

EL tejido empresarial de calidad lo forja la educación empresarial de una sociedad de empleados a una sociedad de empresarios, de una cultura del empleo a una cultura del empresariado. (Ver gráfico 1).

La Educación empresarial, específicamente la transmitida en la universidad genera nuevos empresarios, mediante la creación de una cultura de empresa, en donde el ser empresario no se percibe como fracaso, la familia, el entorno educativo y el entorno general incluyendo el Estado, premia al estudiante emprendedor.

Estos nuevos empresarios de base universitaria EBU, que crean sus empresas, en la mayoría de los casos de supervivencia, 
GRÁFICO 1

Desarrollo económico y educación empresarial

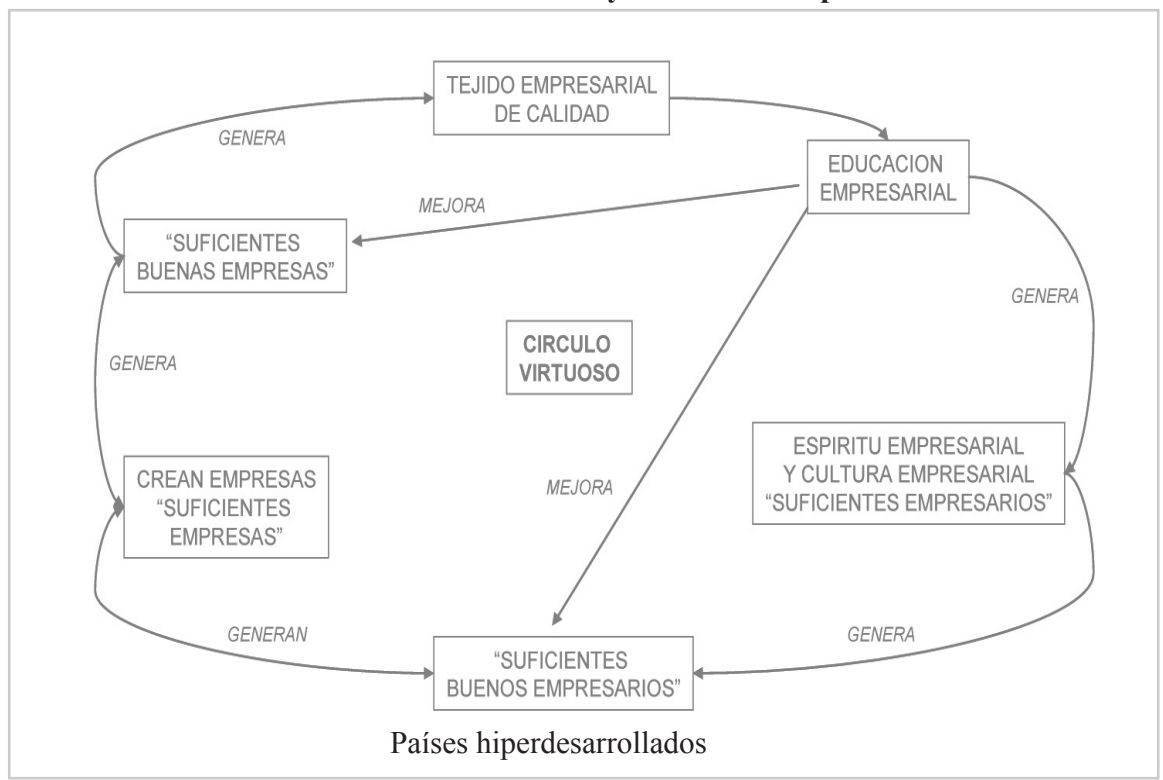

Fuente. Elaboración del autor

necesitan habilidades empresariales, es decir, deben convertirse en mejores gerentes de sus empresas ya creadas, esto con el objetivo de hacerlas crecer y que sean generadoras de empleo y factores claves de éxito. Para lograr este objetivo estos empresarios EBU, retornan a la universidad ya sea a programas formales o de extensión y surgen esas tan anheladas competencias de gerencia profesional. El resultado son gerentes profesionales, buenos empresarios, que desarrollan y transforman empresas de supervivencia en pequeñas y medianas empresas sólidas y competitivas, es decir, suficientes nuevas empresas que fortalecen el tejido empresarial. La universidad de nuevo mediante la investigación aporta a su crecimiento convirtiéndolas en multinacionales tornándose en suficientes buenas empresas. Así como describe el modelo, el tejido empresarial se forja y se fortalece mediante la educación empresarial no solo vista como la motivación y puesta en marcha de empresas, sino como toda la cadena de formación de nuevas empresas, hasta que estas se tornan en empresas multinacionales.

La propuesta de modelo nace en la universidad, como ente generador de conocimiento en la formación empresarial y que hoy a diferencia de la universidad de siglos pasados debe tomar responsabilidad activa en el desarrollo económico y social de los pueblos. 


\section{Educación EMPRESARIAL}

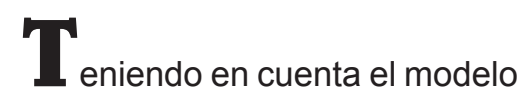
propuesto, la educación empresarial en es la base del cambio cultural y de la construcción de un tejido empresarial de calidad para el desarrollo. Esta es una preocupación en algunas universidades que han tomado la responsabilidad social y de formación más allá de educar y formar personas competentes e idóneas para ocupar cargos.
Diversos estudios e investigadores a nivel internacional donde se destacan, Gibb (2004), Kuratko (2004), Solomon \& Doffy (2002) y Vesper \& Mc Mullen ( 1998), Varela (2000) se orientan a una formación diferente: a formar y educar personas con espíritu empresarial, de tal manera que al finalizar sus estudios universitarios, creen empresas y por esta vía generen empleo, en conclusión aporten al desarrollo económico y social de los pueblos.

\subsection{Educación empresarial como estrategia de creación de nuevas empresas}

Esta ha demostrado a través del tiempo y principalmente desde los años de crísis en los 70 's, ser una buena estrategia para el fomento de creación de nuevas empresas (Fontela y Guzmán, 2004, Rodríguez 2004). El estudio del emprendimiento, entrepreneurship, como ciencia, es relativamente nueva (Veciana 1999). Para algunos investigadores norteamericanos este tópico de investigación ya es un tema maduro (Kuratko 2005); aún así ha dado pie para que investigadores y universidades hayan adoptado dichos estudios para fortalecer $y$ direccionar los currículos hacia la formación empresarial, como vía para su crecimiento e implementación. Su misma escasa edad, en economías emergentes, ha sucitado diversas orientaciones, generando problemas conceptuales (Guzmán y Liñán 2005) que deben ser clarificados para una correcta y efectiva implementación, en el sistema educativo.

\section{¿Qué debe entenderse como educación empresarial?}

Diversos son los investigadores que han empezado a estudiar este fenómeno. Mclntyre y Roche (1999: P33, Asmore 1990, Liñán y Guzmán 2003, Santos 2005, Birley S y Gibb A.A. 1984, Brockhaus, R.H. 1993, Carsrud, A.L. 1991, Comisión Europea 1999) entre muchos otros. De dichos estudios se ha concluido las diferentes definiciones $y$ orientaciones, no solamente basados en el background de los investigadores sino también en la situación geográfica en donde se desarrolla.

Los aportes al conocimiento en educación empresarial tienen diferencias entre Europa y Estados Unidos principalmente en el aspecto geográfico y desde la orientación en la 
formación de las ciencias que lo investigan, como la Psicología, la Administración y las Ciencias Sociales en general.

Así pues, se perciben orientaciones que afirman que el fenómeno de la creación de empresas se orienta al desarrollo de la personalidad en la corriente de Mcleland y otros, la enseñanza de administración como la vía para la creación de empresas y otros más actuales se orientan al desarrollo de nuevas propuestas basadas en la oportunidad y nuevas tecnologías., Druker 1990. En Latinoamérica la orientación se ha enfocado a seguir modelos principalmente de Estados Unidos desconociendo nuestras raíces culturales que son mas europeas que norteamericanas, esto conlleva ciertas desventajas a la hora de implementar estrategias de formación empresarial. (Trejos y otros 2007).

En este trabajo presentamos un acercamiento a la definición de educación empresarial y sus resultados en la formación de empresarios de base universitaria haciendo un comparativo entre Estados Unidos y Europa, de manera conceptual y empírica, contrastándolo con lo que se ha investigado en América latina también de manera empírica y con base en los estudios de: Vesper K.H, $1992^{1}$, Katz, J.A. 1876-1999², en Estados Unidos. Guzmán, J, Liñán $F, 2005^{3}$ en Europa y los recientes estudios de Verdugo, H., Matiz, F y Crissien J. et all ${ }^{4}$, Varela 2005 y Kantis et all en 2006 en Latinoamérica y Colombia.

El objetivo entonces, es presentar un contraste entre las definiciones y orientaciones de Europa y EEUU. Aprovechando el vació que existe en este tópico en nuestro continente, se espera generar un aporte para América Latina, específicamente para Colombia y la presentación de un modelo propuesto para la formación empresarial en la educación superior como forjadores de una nueva raza de empresarios; los empresarios de base universitaria como creadores de empresas, empleos y por ende desarrollo económico, además de los modelos de educación empresarial otro aporte será fortalecer la teoría institucional y de redes en términos de cómo realmente la creación de empresas incide en el desarrollo económico de economías emergentes. (West III, Bamford, Marsden 2008.)

1 VESPER, K.H, 1982 "Research on Education for entrepreneurship" Encyclopedia of entrepreneurship.

2 KATZ, J.A. The Crhonology and Intellectual Trajectory of American entrepreneurship Education 1876-1999, Journal of Business Venturing v. p 283-300, 2003

3 GUZMAN Joaquin, LIÑAN Francisco, Evolucion de la educacion empresarial en Estados Unidos y Europa; su papel como instrumento de desarrollo, Revista Economía Mundial, 122005 149-171

4 BERDUGO, H., MATIZ, F y CRISSIEN J. Formación de Empresarios, Emprendimiento y Creación de Empresas, Universidad EAN 2006 


\subsection{Definición de educación empresarial}

Los más sencillos acercamientos hacia la definición de este punto son los que la describen como la formación para la creación de empresas.

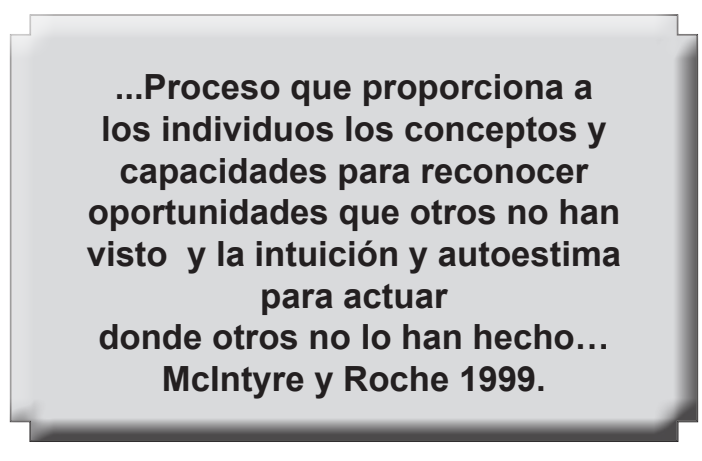

El Consortium for Entrepreneurship Education define como: un modelo de aprendizaje permanente a lo largo de toda la vida del hombre (Ashmore, 1990).

También existen orientaciones no direccionadas específicamente a la definición de la misma sino mas orientadas a la efectiva creación de empresas, para lo cual se fundamentan en las diferentes fases que esta incluye, como es el caso de la Comisión Europea.

Basados en el estudio científico de estos y otros conceptos, optamos por acoger la definición de Liñán (2004: 163) que plantea: ...educación empresarial es el conjunto de todas las actividades de formación reglada o no reglada que intentan desarrollar en los participantes la intención de realizar comportamientos empresariales 0 algunos elementos que influyen sobre esta intención, como el conocimiento empresarial, la deseabilidad de la actividad empresarial o su factibilidad...

Para mayor claridad vale la pena anotar que educación reglada es aquella que está regulada por el gobierno, como en Colombia la Educación primaria y media superior (Bachillerato); en donde los currículos son diseñados y regulados en gran medida por el estado. En el caso de la Educación Superior, es decir la Universidad a pesar de estar regulada, el estado tiene una responsabilidad menor en los aspectos de diseño curricular, y existe el principio de autonomía de la Universidad, lo que significa que las universidades pueden tener libertad en el diseño de programas formales y no formales de educación empresarial. La educación no reglada es aquella que no conduce a título.

Basados en esta definición y con la propuesta del modelo en mente, la universidad como institución debe estar orientada como ente formador de individuos hacia: 
- Desarrollo de comportamientos, competencias emprendedoras.

- Fomentar el espíritu emprendedor y empresarial.

- Fomentar a deseabilidad de ser empresario.

- Formar técnicamente para maximizar la percepción de factibilidad de crear y consolidar nuevas empresas.

Esto como primera opción en la formación universitaria a diferencia de los aspectos modernos que la plantean para ser empleados de empresas ya creadas y como consecuencia de la no empleabilidad causada por la sobreoferta de profesionales, lo que se conoce como el efecto schumpeter, muchos de los profesionales universitarios optan por la creación de autoempleo como única salida. Basados en los problemas conceptuales es importante llegar a una definición clara referente al significado de educación empresarial en general y específico, referente a la responsabilidad de la universidad dentro del marco del sistema educativo de un país para el logro del objetivo que pensamos es el esencial de la universidad; no solo motivar hacia el empresariado, sino la creación efectiva de empresas. Esto es importante anotarlo puesto que de manera cultural en Latinoamérica específicamente el factor de motivación o sensibilización hacia el ser empresario toma visos de mayor importancia que en culturas como la estadounidense en donde de manera natural el deseo de ser empresario es inherente a los individuos (american dream, be your own boss, work your own hours) a diferencia de las culturas europeas, en su mayoría, y la latina en donde el ser empleado de manera cultural es la estrategia profesional por excelencia.

\subsection{Orientaciones de la educación empresarial}

La anterior conceptualización facilita la orientación que no solo en la Universidad se da a dicho tópico. Teniendo en cuenta que esta formación ha demostrado ser una buena estrategia para coadyuvar al desarrollo empresarial, diversas fuentes han empezado a fomentar la creación de empresas, no solo como fuente de empleo sino al desarrollo empresarial. Bajo estos parámetros la universidad ha tomado partido de ciertas estrategias pedagógicas y orientaciones para la formación empresarial.

En el siguiente cuadro, de construcción propia basado en los estudios citados, se describen las diferentes orientaciones de manera general, y su público objetivo , características y resultados esperados. 


\section{EDUCACIÓN EMPRESARIAL CARACTERÍSTICAS}

\begin{tabular}{|c|c|c|c|}
\hline & PÚBLICO OBJETIVO & CARACTERÍSTICAS & $\begin{array}{c}\text { RESULTADO } \\
\text { ESPERADO }\end{array}$ \\
\hline $\begin{array}{l}\text { SENSIBILIZACIÓN } \\
\text { SOBRE EL } \\
\text { EMPRESARIO Y } \\
\text { EMPRESARIADO }\end{array}$ & $\begin{array}{l}\text { Poblaciones vulnerables, } \\
\text { desplazados, madres cabeza } \\
\text { de familia, estudiantes } \\
\text { de los diferentes niveles } \\
\text { desempleados, etc. }\end{array}$ & $\begin{array}{l}\text { Aclaración de qué es una empresa } \\
\text { y sus característica. Qué es un } \\
\text { empresario, qué habilidades, } \\
\text { conductas y motivaciones tiene, } \\
\text { características de ser empresario } \\
\text { como desarrollo profesional y } \\
\text { autoempleo }\end{array}$ & $\begin{array}{l}\text { Optar a SER EMPRESARIO } \\
\text { como proyecto de vida profe- } \\
\text { sional y opción de supervivencia } \\
\text { económica }\end{array}$ \\
\hline $\begin{array}{c}\text { FORMACIÓN PARA } \\
\text { LA CREACIÓN DE } \\
\text { EMPRESAS }\end{array}$ & $\begin{array}{l}\text { Individuos conconocimientos } \\
\text { técnicos de artes u oficios } \\
\text { además de formación uni- } \\
\text { versitaria o técnica con una } \\
\text { orientación a la autonomía } \\
\text { e independencia...ya están } \\
\text { sensibilizados y motivados a } \\
\text { ser empresarios. }\end{array}$ & $\begin{array}{l}\text { Pasos para la creación efectiva de } \\
\text { empresas, tradicionalmente en los } \\
\text { pasos del plan de negocios, fuentes } \\
\text { de financiación, identificación de } \\
\text { ideas de negocios, califi-cación } \\
\text { y cuantificación de mercados, } \\
\text { diseño administrativo y técnico de } \\
\text { empresas, evaluación financiera de } \\
\text { proyectos y fuentes de financiación }\end{array}$ & $\begin{array}{l}\text { Creación y puesta en mar- } \\
\text { cha de empresas nacientes, } \\
\text { Start ups. }\end{array}$ \\
\hline $\begin{array}{c}\text { FORMACIÓN PARA } \\
\text { EL DINAMISMO } \\
\text { EMPRESARIAL }\end{array}$ & $\begin{array}{l}\text { Autompleados y propietarios } \\
\text { de famiempresas, micro, mini, } \\
\text { pequeñas y medianas empresas, } \\
\text { empresarios en acto. }\end{array}$ & $\begin{array}{l}\text { En muchos casos primero se } \\
\text { orienta a formalizar la empresa } \\
\text { en términos legales. orientación } \\
\text { a la administración de la empresa } \\
\text { desde el punto de vista formal, } \\
\text { orientación a la motivación y sen- } \\
\text { sibilización de la necesidad de } \\
\text { organización, gestión y crecimiento } \\
\text { de la empresa como fuente de } \\
\text { estabilidad personal, familiar y de } \\
\text { impacto social y económico }\end{array}$ & $\begin{array}{l}\text { Formalización y crecimiento } \\
\text { de empresas nacientes a } \\
\text { pequeñas y medianas empre- } \\
\text { sas con potencial. }\end{array}$ \\
\hline $\begin{array}{c}\text { FORMACIÓN } \\
\text { CONTINUA PARA } \\
\text { EMPRESARIOS EN } \\
\text { ACTO }\end{array}$ & $\begin{array}{l}\text { Autoempleados y propietarios } \\
\text { de famiempresas, micro, mini, } \\
\text { pequeñas y medianas empresas, } \\
\text { empresarios en acto además de } \\
\text { empresas mipymes con potencial } \\
\text { de crecimiento. }\end{array}$ & $\begin{array}{l}\text { Formación en Administración } \\
\text { y Gerencia de empresas para } \\
\text { la rentabilidad y crecimiento, } \\
\text { desde la supervivencia hasta } \\
\text { el crecimiento estratégico, } \\
\text { además de las características } \\
\text { especiales como empresas } \\
\text { de familia hasta constituirse } \\
\text { en multinacionales interna- } \\
\text { cionalizadas. Técnicas y } \\
\text { modelos gerenciales especí- } \\
\text { ficamente lo denominado } \\
\text { como BBP Best Business } \\
\text { Practices o mejores prácticas } \\
\text { de negocios }\end{array}$ & $\begin{array}{l}\text { Empresas fortalecidas, con } \\
\text { potencial internacional que } \\
\text { aportan de manera importante } \\
\text { al fortalecimiento del tejido } \\
\text { empresarial y al desarrollo } \\
\text { económico, generando valor } \\
\text { agregado y desarrollo social }\end{array}$ \\
\hline
\end{tabular}

Fuente. Elaboración del autor. 


\subsubsection{Educación empresarial en los Estados Unidos}

La orientación, en primer lugar y de manera amplia,sedirigehacialacreacióndeempresas. Aun cuando no se puede generalizar, podría decirse basado en los estudios de Gibb (1993), Katz (2003 ) y otros investigadores, que en los estados Unidos es donde nace la formación empresarial, tal vez por cuanto Schumpeter se radicó allí. La universidad de Harvard fue al parecer la primera universidad en donde se inició el proceso de formación de empresarios, después de la segunda guerra mundial, específicamente en 1947, Miles Mace estableció una asignatura optativa en el Máster en Administración de Empresas denominada gestión de nuevas empresas, en donde, según Katz, participaron aproximadamente 188 de los 600 estudiantes del máster. En la actualidad, este país es el que concentra mayor número de centros de investigación en el tema, mayor número de universidades que plantean la formación empresarial como pilar pedagógico y en definitiva mayor número de empresarios de base universitaria, en donde algunas de las universidades más reconocidas por el hecho son: Babson College, Columbia University, Duke University, Boston University y Harvard, entre otras citadas por Versper (1992). De hecho es en los Estados Unidos donde se plantea que este tema de estudio científico se encuentra en estado de madurez.

...In 1980, fewer than 20 universities and colleges offered courses in entrepreneurship, while today more than 1,600 universities have at least one course in entrepreneurship (Solomon, G.T.Duffy, S., \& Tarabishy, A. 2002).

In fact, the growth rate of entrepreneurship among colleges and universities in the U.S. is nothing short of phenomenal. Katz (2006) argues that the growth continues as we see entrepreneurship courses emerging in the arts, engineering, life sciences, and the liberal arts. Nonetheless, in spite of the fact that entrepreneurship is reaching a state of maturity(Katz, 2006), we continue to debate the definition of entrepreneurship (Fiet, 2001a and 2001b) and the place of entrepreneurship education within the academe (Kuratko, 2003; Katz, 2006). The continued debates suggest that entrepreneurship education is a maturing, yet highly fragmented field of study. In fact, Fiet (2001) argues that the literature on entrepreneurship education is still in a developmental stage. For this reason, we take the view that valuable lessons may be learned from the experiences of successful entrepreneurship programs.

Journal of Entrepreneurship Education, Volume 10, 2007 
La educación empresarial en los Estados Unidos es pues tema de debate, aún en la definición de empresario. Lo que si es un hecho es que los esfuerzos en Estados Unidos se ven recompensados en terminos de:

- Número de universidades con filosofía empresarial.

- Número de programas de pregrado y posgrado específicamente direccionados a la formación de empresarios de base universitaria.

- Generación de claustro docente con el tema de la creación de empresas como tema de estudio y de investigaciondocencia.

- Grupos,centroseinstitutosdeinvestigación orientados al entrepreneurship.

- Journals académicos dedicados al tema

- Congresos dedicados al tema.

- Programas de doctorado con el entrepreneurship como tópico central.

\subsubsection{Educación empresarial en Europa}

Según Santos y Liñán (2005), la orientación de la formación empresarial en la universidades es mas modesta y jóven que en los EEUU. Esta data de los años setenta. En el Reino Unido en 1975, cuando se realizó la primera reunión de la asociación de educación sobre pequeñas empresas y en Francia en 1978, con la Hautes Etudes Comerciales. Han sido muchoslosesfuerzos delaregiónenformalizar asociaciones y grupos de investigación en el tema, los cuales empiezan a dar muestras de fortalecimiento, pero la misma grandeza de la región y las diferencias culturales no permiten tipificar la formación empresarial de manera específica, lo que genera grandes oportunidades de investigación; así mismo, en Europa a diferencia de Estados Unidos no existen hasta el momento, rankings de universidades con formación empresarial como sí aparecen en los Estados Unidos; en Latinoamérica la situación no es diferente, de hecho es más precaria en términos de investigación.

Como lo plantean Santos y Liñán, las diferencias en la formación empresarial entre Europa y Estados Unidos es notable. Los aspectos culturales generan orientaciones diferentes. Se podría aseverar que la educación empresarial en Europa se centra mas en la sensibilización hacia el empresariado. De nuevo no se puede generalizar, pero parecería ser que la misma necesidad percibida, orienta la formación hacia el conocer la profesión de empresario y la carrera empresarial como proyecto de vida, como factor mas preponderante entre los centros educativos estudiados por los nombrados autores. Los programas para la formación efectiva de empresas ocuparon el segundo lugar entre los cerca de 110 centros que se investigaron con un $12 \%$.

En conclusión la formación empresarial en Europa se concentra en la formación para el fomento de una cultura empresarial.

\subsubsection{Educación empresarial en Latinoamérica}

En Latinoamérica, los estudios que más se han acercado a un estudio formal del tema, son los de Kantis y otros (2002) y los del proyecto Tunning, en donde se concentraron en lo relacionado con los procesos de 
creación y desarrollo de nuevas empresas en Latinoamérica y Asia. En el estudio Tunning se evaluó a los egresados de universidades referentes a las competencias por ellos adquiridas durante su formación dentro de las cuales se incluyeron las referentes al espíritu emprendedor y capacidad para la creación de empresas. Los resultados de estos estudios no son para nada halagadores para la universidad.

En el estudio Tunning se puede concluir que la universidad en un mínimo porcentaje aportó para generar las habilidades y competencias necesarias para la carrera empresarial.

En el estudio de Kantis se concluyó que la universidad no fomentó el deseo de ser empresario, mientras que la experiencia fue el factor que más motivo a crear empresa. Así mismo se expresó, que en un alto porcentaje la universidad aportó conocimientos técnicos para la creación y puesta en marcha.

\subsubsection{Educación empresarial en Colombia}

Aún cuando la educación empresarial en Colombia se plantea de manera generalizada en las universidades y se evalúan formalmente como ha sido en este caso, se halló que en la mayoría de los casos no se encuentra nada mas que el slogan. En la práctica no se adelantan ningún tipo de esfuerzos para educar o formar empresarios de base universitaria EBU. Así mismo, el estudio formal y científico de este tema de estudio es supremamente naciente, es decir, es totalmente nuevo.
El estudio más reciente en términos de educación empresarial es el realizado por la Universidad EAN: Formación de Empresarios Emprendimiento y Creación de Empresas (Berdugo, et.al,2006); en donde se presenta un estudio de la educación empresarial en Colombia, que también incluye casos de programas de emprendimiento en diferenes universidades; estudios de estudiantes y egresados. Como conclusión de este estudio se puede plantear que la educación empresarial en Colombia como tópico de investigación es muy nueva, aún así la formación para empresarios es un tema que tiene ya historia en el País. Desde la Escuela de Minas en Medellin, que formó los primeros empresarios y más grandes empresarios de hoy, hasta las universidades pioneras en el tema en Colombia como la Universidad EAN en los años 60 y el ICESI en Cali, en la misma década. La creación de la Fundación Corona, como ente formador y apoyador de nuevos empresarios y ya en la modernidad, universidades como Los ANDES y del Norte de Barranquilla, entre otras.

La educación empresarial en Colombia históricamente se ha concentrado en las carreras o programas de formación en administración de empresas. La orientación de la misma ha sido a la generación de habilidades de administración y de allí la motivación a la creación de empresas. Desde el punto de vista de estudio científico se pueden anotar estudios orientados a investigar la situación ocupacional de los egresados de dichos programas y sus características 
como creadores de empresas; autores como: Crissien 2000, Bonnet y Jiménez (1991), Cortes, Berdugo y Romero (2005), EAN (2004), Álvarez y Calixto (2004), Palomares (1985) y Varela y Jimenez (1994) han aportado al tema formal científico de la formación empresarial.

Finalmente, el libro denominado Universidad EAN 40 años de emprendimiento, hace una reseña histórica de los últimos años de la Universidad en términos de la orientación de la formación de empresarios de base universitaria partiendo de la enseñanza de la administración hasta los días actuales del desarrollo del modelo de formación empresrial denominado EANTEC.
Para este trabajo de investigación, inicialmente se analizaron de manera formal y empírica tres Universidades latinoamericanas, (EAN, ICESI,TEC DE MONTERREY) las cuales enmarcamos dentro del concepto de educación empresarial universitaria; aquellos esfuerzos que incluyen las diferentes orientaciones aquí descritas, es decir, desde la sensibilización hasta la formación contínua a empresarios en acto. Por tanto, de manera general casi todas las universidades latinoamericanas han optado por el slogan de formación empresarial como diferenciación mercadológica, adoptando tal vez la orientación Norteamenricana.

La recolección de datos en 50 universidades Bogotanas, arrojaron una diferencia en lo que se denomina educación empresarial de manera científica.

\subsection{Universidades con educación empresarial}

Este estudio da la posibilidad de proponer una clasificación de universidades con formación empresarial así:

\section{- Nivel uno}

Universidades que de manera informal plantean el emprendimiento como parte de su modelo pedagógico pero que no se evidencia en ninguna parte del currículo ni de los servicios complementarios de las mismas. En este nivel se enmarcan que ofertan programas de Administración de Empresas e Ingeniería Industrial. De manerageneral todas aquellas que tienen en sus currículos programas de formación en ciencias económicas y empresariales y que definen formación empresarial en los aspectos enmarcados dentro de estas ciencias pero que no aportan a la sensibilización hacia la empresa y el empresariado como orientación profesional, en Colombia 176, (Calixto 2005)

\section{- Nivel dos}

Universidades como las anteriores, pero con la diferencia que en ellas existen uno o varios profesores con la filosofía del emprendimiento y que de manera formal o informal imparten en sus cursos un sello emprendedor en el marco de la libertad de cátedra. Estos profesores muchas veces son tildados de locos en los claustros docentes 
universitarios, pero aun así un buen número de nuevos empresarios de base universitaria afirmaron que su decisión de ser empresarios fue motivada por un profesor en especial durante el curso de su formación. (Matiz 2005).

\section{- Nivel tres}

Universidades que cuentan de manera complementaria, como servicio de bienestar universitario el apoyar a estudiantes que estén interesados en la creación de empresas. En este nivel podrían incluirse las que cuentan con centros para este tipo de apoyo, pero que no están inmersos en el currículo; las que tienen convenio con el Servicio Nacional de Aprendizaje SENA, y se han caracterizado como universidades con unidades de emprendimiento; así mismo en esta distribución se podrían inlcuir las que han adoptado algún modelo ya generado como el modelo de tranferencia del TEc de Monterrey o el modelo de Jóvenes con Empresas del BID.

\section{- Nivel cuatro}

Universidades que como primera medida en su filosofía, en los planes Educativos Institucionales PEI demuestran su compromiso con la formación de emprendedores -empresarios. Estos cuentan con programas de apoyo y centros especializados para el fomento del espíritu empresarial y creación de empresas y además esta orientación está inmersa de manera transversal en el currículo; por lo general estas universidades han desarrollado modelos propios de enseñanza del emprendimiento o han implementado programas de transferencia del conocimiento. Así mismo, dentro de las metodologías incluyen los modelos de rol y los concursos de planes de negocios, cuentan con vínculos interinstitucionales para la financiación y puesta en marcha de los proyectos. Es importante anotar que una característica de este tipo de instituciones es que en los proyectos finales de graduación o usualmente denominados "Tesis de grado" cuentan con el formato de creación de empresas como una alternativa.

\section{- Nivel cinco}

Universidades como las anteriores, pero que han desarrollado modelos de incubación, es decir, el más alto nivel de universidad emprendedora; aquella que se ha comprometido en la sensibilización por la vía curricular,y que capacitan a los estudiantes para la efectiva creación de empresas y los incuban para la puesta en marcha, de tal forma que efectivamente se crea empresa. Así mismo, con sus egresados existen programas de dinamización de las empresas ya creadas por la vía de la formación contínua para este objetivo y finalmente, cuentan con programas de formación contínua para empresarios en temas especializados en gerencia profesional y mejores prácticas de negocios. De otra parte, la misma universidad es una empresa del conocimiento generando consultoría y transfiriendo sus modelos de formación a otras, a nivel nacional e internacional.

Como conclusion final de este avance de estudio se aporta lo siguiente:

- Si se quiere tener un tejido empresarial de calidad que genere desarrollo económico, la universidad pública y privada debe comprometerse más con la eduacion empresarial. 
- En el seno de la universidad se deben fortalecer los campos investigativos en Entrepreneurship.

- Se debe fomentar mas la creacion de grupos de centros y de institutos de investigacion en entrepreneurship.

- Se debe profundizar en el studio del entrepreneurship mas desde el punto de vista de los modelos pedagogicos para formasr EBU que los resultados, perfiles y caracterizacion de empresarios Colombianos y Latinoamericanos.

- Es muy importante darnos cuenta como region, Latinoamérica, que tenemos caracteristicas culturales, socials, tecnologicas, y politicas entre otras que nos hacen unicos y amerita investigacion propia.

- Finalmente, el campo del entrepreneurship y de la educacion empresarial en latinoamerica es supremamente joven desde el punto de vista científico.

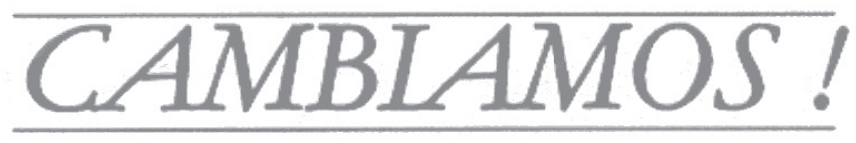

Correos

de Colombia

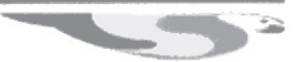

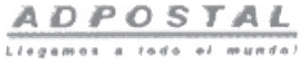

Pensando en ofrecerle el mejor servicio

Nuestras Líneas de Atención al Cliente

4298487 - 2633484 - 2956896

$018000111210 / 111313$

Fax: 4163026

Subgerencia de Mercadeo

3340304

División de Mercadeo Regional DC 4297320

www.adpostal.gov.co 


\section{Bibliografía}

Audretsc,D.B., CarreeM.A., ThurikA.R.,(2002)“DoesEntrepreneurshipreduce Unemployment? Tinbergen Institute. Erasmus University, Rotterdam TI 2001-074/3.

Bruton Gary D., Ahlstrom David, Obloj K.(2008). Entrepreneurship in Emerging Economies, ET\&P, Vol 32 No 1 January 2008.

Crissien J, Pérez R. (2007) EAN 40 años de emprendimiento, EAN. Compilador.

Crissien J, Espíritu empresarial como estrategia de competitividad y desarrollo económico en: Revista EAN No 592007.

Crissien J. (2006): Incidencia de los modelos de rol y formación universitaria empresarial como detonadores en la creación de empresas en Colombia. Proyecto de investigación, Doctorado en ciencias empresariales, Universidad Antonio de Nebrija, Madrid España. Paper de trabajo.

Druker P. (1996). La innovación y el empresario Innovador, Cali, Colombia: Norma.

Gardner W.B. (1990): What are we talking About when we talk about Entrepreneurship ? Journal of Business Ventures , p.p 5-28

Gibb, A. (2004).Creating Conductiva Environment for learning an entrepreneruship living with, delaing with creating and enjoying uncertainty and complexity, 14th annual International Conference, University of Napoli, Federico 11 ( Italy) Napoles.

Gibb A. (2005): Creating the entrepreneurial university worldwide. do we need a wholly different model of entrepreneurship?. Dicussion Paper ponencia,CIELA 4ta Conferencia de investigacion en entrepreneurship en Latinoamérica, Cali Colombia.

González Domínguez, F.J. (2004) Tesis Doctoral Incidencia del Marco Institucional en la capacidad emprendedora de los jóvenes empresarios de Andalucía, Universidad de Sevilla.

Guzman J. y Liñan F.( 2005): " Evolución de la educación empresarial en Estados Unidos y Europa: Su papel como instrumento de desarrollo". Revista de economía Mundial, 12, 2005, 149-171.

Kantis H., (2005): Desarrollo emprendedor, América Latina y la experiencia internacional. Banco Interamericano de Desarrollo, BID.

Kirzner, I.M, (1979): Perception, Oportunity and profit. Studies in the theory of entrepreneurship. The University of Chicago Press, Chicago. 
Kuratko, D.F. (2004): Entrepreneurship Educatión in the 21 Century: From legitimitation to leadership, USASBA National Conference.

Pappin R. (2005): Cómo favorecer el emprendimiento en América Latina. Disscution paper, Ponencia CIELA 4ta Conferencia de investigacion en entrepreneurship en Latinoamérica, Cali Colombia.

Ronstadt, R.C. (1985): Training Potential Entrepreneurs. Entrepreneurship: What it is and how to theach it P.Pp 191-204.

Shapero, A. (1984): The entrepreneurial Event in. KENT C.A. (Ed) The environment for entrepreneurship Lexintong Book, D.C. Health.

Shane S; Venkataraman, S.(2000): THe promise of entrepreneurship a field of research, Academy of management Review, vol 25. No. 1.

Solomon T. Duffy S.(2002): The state of Entrepreneurship Education in the U.S. A Nationwide Survey and analysis, International Journal of entrepreneurship Education, P.65-68.

Schumpeter J.A. (1982): Historia del análisis económico.Madrid: segunda ed.Arial.

Timmons J.(1999): New Venture Creation, Entrepreneruship for the 21st Centrury. IRWIN, Mc Graw Hill. Boston.

Varela R. (2001): La cultura empresarial como estrategia de desarrollo para América Latina. Revista Icesi. Universidad ICESI, Cali Colombia.

Varela R. (2003): Hacia una universidad con espíritu empresarial. Disscution paper, Ponencia, II Conferencia de investigacion en entreprenership en Latinoamérica, Viña del Mar Chile.

Varela R., Bedoya O., Modelo conceptual de desarrollo empresarial basado en competencias, ICESI; Colombia.

Varela R.(2001): Innovación empresarial; arte y ciencia de la creación de nuevas empresas. Pearson Education, Bogotá Colombia.

Veciana, Vergés, J.M. (1999): "Creación de Empresas como programa de investigación Científica" Revista Europea de Dirección y Economía de la Empresa, Vol 8. No.3 pp 11-36.

Veciana, Vergés, J.M. (1979): ¿Emprendedor o empresario? Innovando. Boletín del Centro de Desarrollo del Espíritu Empresarial Universidad ICESI Colombia. No. 17, diciembre. 


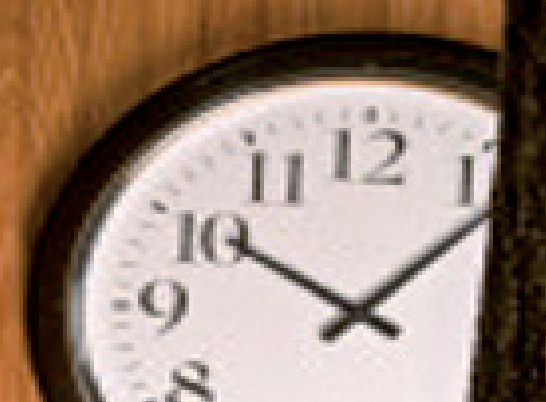

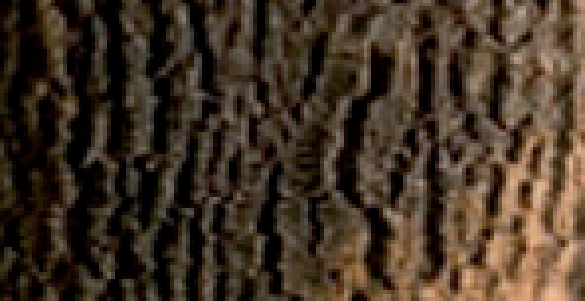

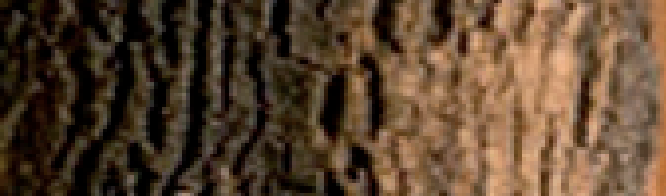
s.

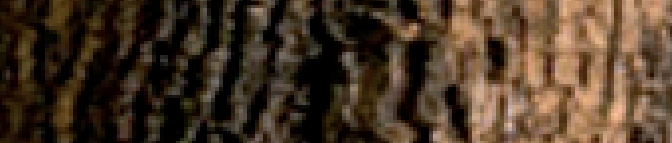

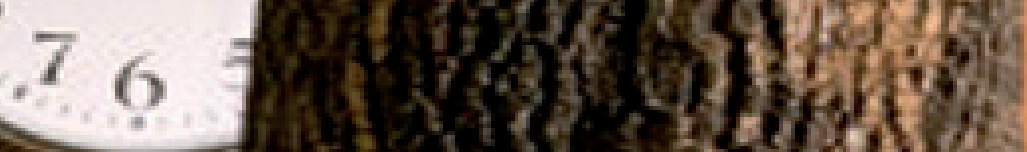

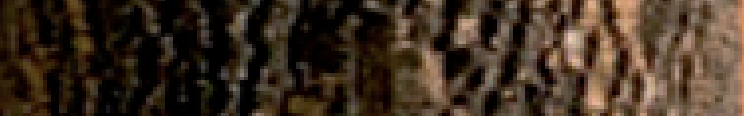

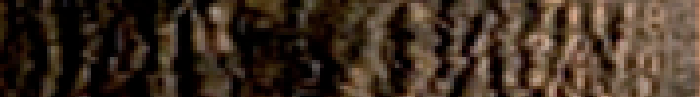

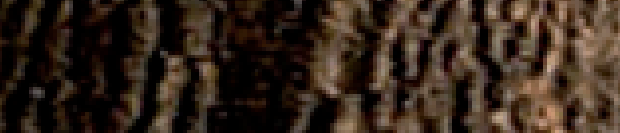

Hays

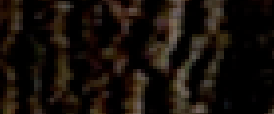

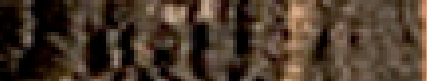

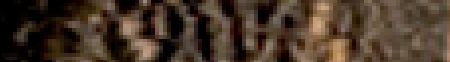

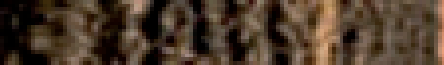

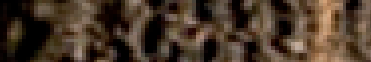

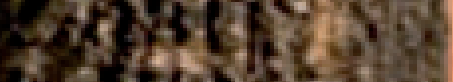
3. 3 and ofis 1)isty a) 8 \%

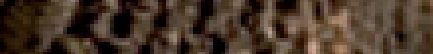

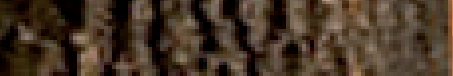
sotising (xis)

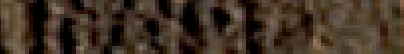

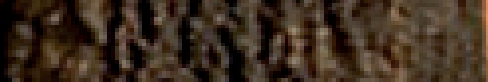

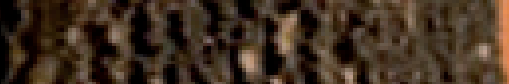

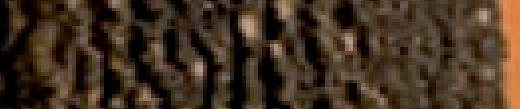

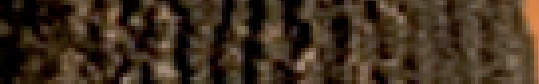

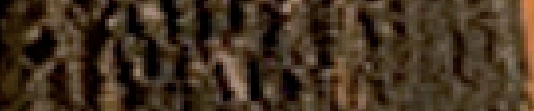

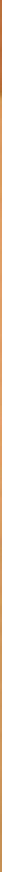

